\title{
Synchronous Genital Tract Neoplasms
}

\author{
Maj JRA Duckett \\ $M B, C h B, M R C O G, R A M C(V)$ \\ Locum Consultant Obstetrics and Gynaecology
}

City Hospital NHS Trust, Dudley Road, Birmingham B18 7QH

SUMMARY: Synchronous genital tract neoplasms constitute a more common clinical problem than would be generally expected. This case focuses on mixed mullerian tumours and postulates a mechanism for an increased incidence found associated with synchronous genital tract neoplasms.

\section{Introduction}

Mixed mullerian tumours (MMTs) are an uncommon uterine malignancy but usually present in a similar way to other uterine malignancies with vaginal bleeding. MMTs account for $2 \%$ of endometrial malignancies (1). The exact diagnosis is usually made at the histopathological examination of the uterus. Histologically MMTs have both epithelial and mesenchymal elements and may contain endometrial stroma, smooth muscle, cartilage, bone, fat, neuronal elements etc. Synchronous genital tract neoplasms are also an unusual finding. However, the two conditions may be linked by the theory of the secondary mullerian system. There is the possibility that when embryologically immature tissue is subjected to a carcinogenic stimulus, it may result in tumours with varying cell lines. Hence a carcinogenic stimulus affecting the genital tract may be expected to cause an increased number of MMTs and other synchronous genital tract tumours.

\section{Case Report}

An 82 year old female presented to casualty having fallen out of bed. She sustained a scalp laceration involving considerable blood loss. There were no other injuries. The patient was successfully resuscitated with blood and crystalloid.

A history was obtained via her two daughters (her only pregnancies) as she was profoundly deaf. She had non insulin dependent diabetes (controlled with metformin $850 \mathrm{mg}$ ), chronic obstructive airways disease (for which she used beclomethasone 200 micrograms, twice daily) and hypertension (controlled with xipamide $20 \mathrm{mg}$ ). She had long term urinary incontinence which had not been investigated and had very poor vision due to bilateral cateracts.

An incidental finding of a full clinical examination was a mass arising out of the right side of the patient's pelvis. Vaginal examination showed a 15 by $20 \mathrm{~cm}$ diameter, smooth, firm, mobile mass separate from the uterus. Rectal examination was normal. Urinalysis showed an E coli urinary tract infection which was treated.

A gynaecological opinion was requested and further questioning revealed a history of post-menopausal bleeding and mild low abdominal pain. She had never had a cervical smear test. The mass was asymptomatic and she had not noted any abdominal swelling, systemic disturbance or weight loss. There were no gastrointestinal symptoms. A transabdominal ultrasound examination showed a large right $20 \mathrm{~cm}$ ovarian tumour with multilocular cystic and solid components. There was no evidence of hepatic metastasis and no hydronephrosis. The uterus was reported as normal with no endometrial thickening. There was no evidence of ascites and the left ovary was normal. Computerized tomography or nuclear magnetic resonance scans were not performed as their findings were unlikely to yield further useful clinical information which would alter the decision for laparotomy.

Any ovarian mass in a post-menopausal woman is suspicious of malignancy. The solid components and multicystic nature of the mass made ovarian malignancy more likely. After consultation with the patient, her relatives and the anaesthetist the patient underwent a laparotomy, total abdominal hysterectomy and bilateral salpingo-oophorectomy. At laparotomy there was a twenty centimeter right ovarian cyst. The uterus was a little bulky but otherwise externally normal. The left ovary, tubes and upper abdominal organs were normal. There was no ascites or evidence of metastasis. The omentum was not clinically involved in the disease process. Peritoneal washings were sent for cytological assessment. There were no palpably enlarged lymph nodes. Histology of the specimen showed cervical, endometrial and ovarian tumours of different histological types. The cervical lesion was a stage 1B adenosquamous carcinoma, whereas the endometrial lesion was a stage 1 malignant mixed mullerian tumour and the ovarian tumour was a benign mucinous cystadenoma. There was no histological involvement of the omentum and the peritoneal washings were negative for malignant cells. Postoperatively the patient made a good recovery but was judged to be unfit for further radiotherapy or chemotherapy.

The patient died 12 months after initial surgery of disseminated carcinomatosis with bowel obstruction. She was considered unfit for palliative surgery shortly prior to her death. A postmortem was not performed.

\section{Discussion}

Synchronous multiple gynaecological neoplasms are rare but are probably found more commonly than by chance alone $(2,3)$. This woman presented with a mass as well as postmenopausal bleeding and the clincial diagnosis suggested a primary ovarian malignancy. The ovarian tumour was subsequently found to be benign. The patient also had other co-existent pathology which remained undetected until the specimen was examined pathologically. This case shows some of the limitations of ultrasound in predicting ovarian malignancy. When investigating postmenopausal bleeding a malignancy is found in $12 \%$ of women (4). Of the women with malignancy, $67 \%$ will be endometrial adenocarcinomas, $17 \%$ will be cervical squamous carcinomas and $17 \%$ will be ovarian carcinomas. The endometrium can be further assessed with ultrasound. An endometrial thickness of $5 \mathrm{~mm}$ or less (on transvaginal scanning) will confidently exclude endometrial malignancy (5). In this woman the endometrium was not thickened but ultrasound assessment by the abdominal route is not as sensitive as with transvaginal scanning. Smear testing is used to screen for squamous cervical intraepithelial neoplasia but is less useful at predicting adenomatous lesions (6). The cervix looked normal macroscopically. However, a smear test may 
have been useful in identifying this woman's adenosquamous lesion due to the squamous component of the tumour. The cervical smear program has not reached all women and women who do present with cervical tumours have often never had a smear.

The place for treating MMTs with radiotherapy or chemotherapy is uncertain. There is a tendency for early haematogenous and lymphatic spread with as many as $45 \%$ of stage 1 uterine MMTs having lymph node metastases (7). This implies that local radiotherapy is unlikely to be beneficial. Eighty percent of stage 1 endometrial adenocarcinomas are cured by simple hysterectomy but the prognosis is very much poorer with MMTs. This is illustrated in the above case.

Eisner et al (8) reported only one case of three synchronous tumours in 3863 patients with female genital malignancies. Interestingly, this involved a mixed mullerian tumour of the ovary. Of four patients with synchronous tumours of the endometrium and cervix, one was associated with a MMT of the endometrium. A study of uterine MMTs by Lotocki et al (9) found a history of cervical cancer in $16 \%$ of cases. MMTs account for only $2 \%$ of endometrial malignancies (1) but the possible increased numbers of multiple gynaecological neoplasms found in the studies of MMTs has not been well documented in the past.

The uterus arises by the fusion of the Mullerian ducts, which are formed from the mesenchyme of the urogential ridge and lined by epithelium derived from the coelomic epithelium. MMTs of the uterus are derived from both these epithelial and mesenchymal elements. Genital tract tissue has an inherent ability to differentiate into different cell lines e.g., teratomas.

The theory of the secondary mullerian system (10) suggest that embryologically similar tissue (as found in the cervix, uterus, tubes, ovaries and peritoneum), simultaneously subjected to hormone influence or carcinogens, may develop synchronous neoplasms. A carcinogenic stimulus affecting the genital tract would affect embryologically primitive cells. Hence the theory of the secondary mullerian system would account for an increased incidence of synchronous and embryologically diverse tumours, e.g., the sychronous mixed mullerian, cervical and ovarian tumours as seen in the case above.

\section{REFERENCES}

1. Chuang JT, Val Vleden DJJ, Graham JB. Carcinosarcoma and mixed mesodermal tumours of the uterine corpus: review of 49 cases. Obstet Gynecol 1970; 35: 769-80.

2. Rose PG, Herterick EE, Boutselis JG, Moeshberger M, SACHS L. Multiple gynaecological primary neoplasms. Am J Obstet Gynecol 1987; 157: 261-267.

3. AXelrod J, Fruchter R, Boyce GJ. Multiple primaries among gynaecological malignancies. Gynecol Oncol 1984; 18: $359-372$.

4. Wagman H. Postmenopausal bleeding. Baillieres Clin Obstet Gynaecol 1988; 2(2):

5. Granberg S, WiKland M, Karlsson B, et al. Endometrial thickness as measured by endovaginal ultrasound for identifying endometrial abnormality. Am J Obstet Gynecol 1991; 164: 47-52.

6. Luesley D. Cervical adenocarcinoma. In: Studd J, ed. Progress in Obstetrics and Gynaecology Volume 7. London: Churchill Livingstone, 1989; 369-387.

7. CHEN SS. Propensity of retroperitoneal lymph node metastasis in patients with stage 1 sarcoma of the uterus. Gynecol Oncol 1989; 32: 215-217.

8. EISNER RF, NIEBERG RK, BEREK JS. Synchronous primary neoplasms of the female reproductive tract. Gynecol Oncol. $1989 ; 33: 335-339$.

9. Lotoki R, Rosenshein NB, Crumbine R, Dillon M, PARMLEY T, WOODRUfF D. Mixed mullerian tumours of the uterus: clinical nad pathologic correlations. Int $J$ Gynecol Obstet 1982; 20: 237-43.

10. Woodruff JD, Soloman D, Sullivant H. Multifocal disease in the upper genital canal. Obstet Gynecol 1985; 65: 695-698. 